\title{
Ground- and excited-state cusp conditions for the pair density
}

\author{
Á. Nagy ${ }^{1}$ and C. Amovilli ${ }^{2}$ \\ ${ }^{1}$ Department of Theoretical Physics, University of Debrecen, H-4010 Debrecen, Hungary \\ ${ }^{2}$ Dipartimento di Chimica e Chimica Industriale, Universitá di Pisa, Via Risorgimento 35, I-56126 Pisa, Italy \\ (Received 21 April 2010; published 22 October 2010)
}

\begin{abstract}
Higher order cusp relations are derived for the pair density of the ground and excited states of atoms, ions, or molecules. Both electron-nucleus and electron-electron coalescences are studied.
\end{abstract}

DOI: 10.1103/PhysRevA.82.042510

PACS number(s): 31.15.E-

\section{INTRODUCTION}

Cusp relations have turned out to be extremely useful in studying exact properties of several quantities such as the wave function, the electron density, and the kinetic energy density. They are associated with the singularity of the Coulomb potential. The nature of the cusp was first studied by Kato [1]. Electron-electron coalescence was studied for the wave function by Kolos and Roothaan [2], Roothaan and Kelly [3], and Pack and Brown [4]. Cusp conditions were derived for the electron density [5], the first-order density matrix [6], the uniform electron gas [7,8], and the density matrices $[9,10]$. Three-particle coalescence was also studied [11-13]. Cusp relations were derived for the density for highly excited states [14]. The curvature of the electron density in the ground state was studied by Esquivel et al. [15,16], and the generalization for excited states was also presented [17]. Nuclear cusp conditions for the energy density were obtained by March et al. [18]. Relations between the derivatives for the many-electron wave functions at the cusp were derived by Rassolov and Chipman [19], and expressions between the derivatives of the densities were also derived for both the ground and the excited states [20].

The pair density has become very important as it is the key quantity of pair density functional theory [21-32]. In this article, cusp relations are presented for the pair density both for the ground and the excited states. These expressions include the first and the third derivatives.

In the following section, the higher order nuclear cusp relations are summarized [20]. In Sec. III, higher order electron-electron cusp relations are derived for the groundand excited-state wave functions. Section IV presents higher order cusp conditions for the pair density. The last section is devoted to illustrative examples and discussions.

\section{HIGHER ORDER NUCLEAR CUSP RELATIONS FOR THE GROUND- AND EXCITED-STATE WAVE FUNCTIONS}

First, the higher order nuclear cusp relations derived earlier [20] are summarized. The total Hamiltonian can be written as

$$
\hat{H}=\hat{H}_{1}+\hat{W}+\hat{G},
$$

where

$$
\hat{H}_{1}=-\frac{1}{2} \nabla_{1}^{2}-\frac{Z_{\alpha}}{r_{1}},
$$

$$
\begin{gathered}
\hat{W}=-\sum_{\beta \neq \alpha} \frac{Z_{\beta}}{\left|\mathbf{r}_{1}-\mathbf{R}_{\beta}\right|}+\sum_{j \neq 1}^{N} \frac{1}{\left|\mathbf{r}_{1}-\mathbf{r}_{j}\right|}, \\
\hat{G}=-\sum_{i=2}^{N} \sum_{\beta \neq \alpha} \frac{Z_{\beta}}{\left|\mathbf{r}_{i}-\mathbf{R}_{\beta}\right|}+\frac{1}{2} \sum_{i=2}^{N} \sum_{j \neq i}^{N} \frac{1}{\left|\mathbf{r}_{i}-\mathbf{r}_{j}\right|} \\
-\frac{1}{2} \sum_{i=2}^{N} \nabla_{i}^{2}-\sum_{j \neq 1}^{N} \frac{Z_{\alpha}}{r_{j}} .
\end{gathered}
$$

We will study the wave function in the vicinity of the nucleus $\alpha$, and the origin of the coordinate system is placed at the nucleus $\alpha$. In this article, only two-particle coalescence is studied. As we consider the case where $\left|\mathbf{r}_{1}\right|=r_{1}$ is small, smaller than $r_{2}$, the following expansion can be used in $W$ :

$$
\frac{1}{\left|\mathbf{r}_{1}-\mathbf{r}_{2}\right|}=\sum_{l, m} \frac{4 \pi}{2 l+1} \frac{r_{1}^{l}}{r_{2}^{l+1}} Y_{l m}^{*}\left(\Omega_{1}\right) Y_{l m}\left(\Omega_{2}\right) .
$$

So the expansion of $W$ has the form

$$
W=\sum_{l, m} r_{1}^{l} W_{l m} Y_{l m}^{*}\left(\Omega_{1}\right)
$$

where $W_{l m}$ depend on $\mathbf{r}_{2}, \ldots, \mathbf{r}_{N}$ but do not depend on $\mathbf{r}_{1}$ :

$$
W_{l m}=-\frac{4 \pi}{2 l+1}\left[\sum_{\beta \neq \alpha} Z_{\beta} \frac{Y_{l m}\left(\Omega_{\beta}\right)}{R_{\beta}^{l+1}}-\sum_{j>1} \frac{Y_{l m}\left(\Omega_{j}\right)}{r_{j}^{l+1}}\right] .
$$

$Y_{l m}$ are the spherical harmonics. The operator $G$ acts only on $\mathbf{r}_{2}, \ldots, \mathbf{r}_{N}$.

The most general expansion of the antisymmetric wave function around the nucleus $\alpha$ is

$$
\Phi=\sum_{l=0}^{\infty} \sum_{m=-l}^{m=l} r_{1}^{l} \chi_{l m}\left(r_{1}, X\right) Y_{l m}\left(\Omega_{1}\right)
$$

where $X$ stands for the coordinates $\sigma_{1}, \mathbf{r}_{2}, \sigma_{2}, \ldots, \mathbf{r}_{N}, \sigma_{N}$. We note in passing that the antisymmetry of the wave function in Eq. (8) is retained only with the full summation over $l$ from zero to infinity.

In certain highly excited states, the spherical average of the derivative of the wave function is zero at a nucleus: $\chi_{00}(0, X)=$ 0 . This is the case where there are no $s$ electrons. To include this case and the even more special cases where

$$
\chi_{\operatorname{lm}}(0, X)=0
$$


$l>0$, we rewrote Eq. (8) as

$$
\begin{aligned}
\Phi= & \sum_{m} r_{1}^{l} \chi_{l m}\left(r_{1}, X\right) Y_{l m}\left(\Omega_{1}\right) \\
& +\sum_{l^{\prime}>l} \sum_{m^{\prime}} r_{1}^{l^{\prime}} \chi_{l^{\prime} m^{\prime}}\left(r_{1}, X\right) Y_{l^{\prime} m^{\prime}}\left(\Omega_{1}\right),
\end{aligned}
$$

where $l$ is the smallest integer for which $\chi_{l m}$ is not zero. In the second term in Eq. (10), the summation should go only for $l^{\prime}>l$. Such highly excited atoms have been observed, for example, the $2 p^{3}{ }^{4} S$ state of a negative $\mathrm{He}$ ion and of $\mathrm{Li} \mathrm{I}$, Be II, B III, and C IV ions [33].

The function $\chi_{l m}$ can be expanded as

$\chi_{l m}(r, X)=a_{l m}^{(0)}(X)+a_{l m}^{(1)}(X) r+a_{l m}^{(2)}(X) r^{2}+a_{l m}^{(3)}(X) r^{3}+\cdots$

Substituting expressions (10), (11), and (6) into the Schrödinger equation $\hat{H} \Phi=E \Phi$, multiplying it with the spherical harmonics $Y_{l m}^{*}\left(\Omega_{1}\right)$, integrating on the polar angles $\Omega_{1}$, and equating the coefficients of $r^{l-1}, r^{l}, r^{l+1}$ separately to zero, we arrive at the system of equations

$$
\begin{gathered}
Z_{\alpha} a_{l m}^{(0)}(X)+(l+1) a_{l m}^{(1)}(X)=0, \\
(2 l+3) a_{l m}^{(2)}(X)+Z_{\alpha} a_{l m}^{(1)}(X)-(\hat{G}+\bar{W}-E) a_{l m}^{(0)}(X)=0,
\end{gathered}
$$

$3(l+2) a_{l m}^{(3)}(X)+Z_{\alpha} a_{l m}^{(2)}(X)-(\hat{G}+\bar{W}-E) a_{l m}^{(1)}(X)=0$,

where $\bar{W}=(4 \pi)^{-1 / 2} W_{00}$ is constant. Combining these equations, we are led to the relations for the terms $a_{l m}$ :

$$
\begin{gathered}
a_{l m}^{(1)}(X)=-\frac{Z_{\alpha}}{l+1} a_{l m}^{(0)}(X), \\
a_{l m}^{(2)}(X)=\frac{1}{2 l+3}\left[\frac{Z_{\alpha}^{2}}{l+1}+\hat{G}+\bar{W}-E\right] a_{l m}^{(0)}(X), \\
a_{l m}^{(3)}(X)=-\frac{Z_{\alpha}}{3(l+1)(l+2)} \\
\times\left[(3 l+4) a_{l m}^{(2)}(X)-\frac{Z_{\alpha}}{l+1} a_{l m}^{(0)}(X)\right] .
\end{gathered}
$$

If $l=0$, the relations of Rassolov and Chipman [19] can be recovered. In the following section, the electron-electron cusp relations are derived.

\section{HIGHER ORDER ELECTRON-ELECTRON CUSP RELATIONS FOR THE GROUND- AND EXCITED-STATE WAVE FUNCTIONS}

Now, we turn to the electron-electron coalescence and derive electron-electron cusp relations. We proceed similarly as we did in the previous section. The same total Hamiltonian can be now be rewritten as

$$
\hat{H}=\hat{H}_{12}+\hat{U}+\hat{K},
$$

where

$$
\hat{H}_{12}=-\nabla_{r_{12}}^{2}+\frac{1}{r_{12}}
$$

$$
\begin{gathered}
\hat{U}=-\sum_{\alpha} Z_{\alpha}\left(\frac{1}{\left|\mathbf{r}_{1}-\mathbf{R}_{\alpha}\right|}+\frac{1}{\left|\mathbf{r}_{2}-\mathbf{R}_{\alpha}\right|}\right)+\sum_{j=3}^{N}\left(\frac{1}{\mathbf{r}_{1 j}}+\frac{1}{\mathbf{r}_{2 j}}\right), \\
\hat{K}=-\frac{1}{4} \nabla_{R}^{2}-\frac{1}{2} \sum_{j=3}^{N} \nabla_{i}^{2}-\sum_{i=3}^{N} \sum_{\alpha} \frac{Z_{\alpha}}{\left|\mathbf{r}_{i}-\mathbf{R}_{\alpha}\right|}+\frac{1}{2} \sum_{\substack{i, j=3 \\
i \neq j}}^{N} \frac{1}{\mathbf{r}_{i j}}, \\
\mathbf{r}_{12}=\mathbf{r}_{1}-\mathbf{r}_{2}, \quad \mathbf{R}=\frac{1}{2}\left(\mathbf{r}_{1}+\mathbf{r}_{2}\right) .
\end{gathered}
$$

Now we expand the wave function and the potential $\hat{U}$ around $r_{12}=0$ :

$$
\begin{gathered}
\Phi=\sum_{l^{\prime} \geqslant l}^{\infty} \sum_{m^{\prime}=-l}^{m^{\prime}=l} r_{12}^{l^{\prime}} \gamma_{l^{\prime} m^{\prime}}\left(r_{12}, X\right) Y_{l^{\prime} m^{\prime}}\left(\Omega_{12}\right) \\
U=\sum_{l^{\prime}, m^{\prime}} r_{12}^{l^{\prime}} U_{l^{\prime} m^{\prime}} Y_{l^{\prime} m^{\prime}}^{*}\left(\Omega_{12}\right)
\end{gathered}
$$

where, in this section, $X$ stands for the coordinates $\sigma_{1}, \sigma_{2}, \mathbf{R}, \mathbf{r}_{2}, \ldots, \mathbf{r}_{N}, s_{N}$ and

$$
\begin{aligned}
U_{l m}= & \frac{4 \pi}{2 l+1}\left(\frac{1}{2}\right)^{l}\left\{\frac { 1 } { 2 } \sum _ { j = 3 } ^ { N } \frac { 1 } { | \mathbf { R } - \mathbf { r } _ { j } | ^ { l + 1 } } \left[Y_{l m}\left(\widehat{\mathbf{r}_{j}-\mathbf{R}}\right)\right.\right. \\
& \left.+Y_{l m}(\widehat{\mathbf{R - r}})\right]-\sum_{\alpha} \frac{1}{\left|\mathbf{R}_{\alpha}-\mathbf{R}\right|^{l+1}}\left[Y_{l m}\left(\widehat{\mathbf{R}_{\alpha}-\mathbf{R}}\right)\right. \\
& \left.\left.+Y_{l m}\left(\widehat{\mathbf{R - r}} \mathbf{r}_{\alpha}\right)\right]\right\} .
\end{aligned}
$$

We note in passing that if we take the ground state or low-lying excited state, then $l=0 ; l \neq 0$ only when we consider an excited state with no $s$ electrons. The function $\gamma_{l m}$ can now be expanded as

$$
\begin{aligned}
\gamma_{l m}\left(r_{12}, X\right)= & b_{l m}^{(0)}(X)+b_{l m}^{(1)}(X) r_{12}+b_{l m}^{(2)}(X) r_{12}^{2} \\
& +b_{l m}^{(3)}(X) r_{12}^{3}+\cdots
\end{aligned}
$$

Substituting expressions (23), (26), and (25) into the Schrödinger eguation, multiplying it with the spherical harmonics $Y_{l m}^{*}\left(\Omega_{12}\right)$, integrating on the polar angles $\Omega_{12}$, and equating the coefficients of $r_{12}^{l-1}, r_{12}^{l}, r_{12}^{l+1}$ separately to zero, we arrive at the relations

$$
\begin{gathered}
b_{l m}^{(1)}(X)=\frac{1}{2(l+1)} b_{l m}^{(0)}(X), \\
b_{l m}^{(2)}(X)=\frac{1}{2 l+3}\left[\frac{1}{4(l+1)}+\frac{1}{2}(\hat{K}+\bar{U}-E)\right] b_{l m}^{(0)}(X),
\end{gathered}
$$

$$
\begin{aligned}
& b_{l m}^{(3)}(X) \\
& \quad=\frac{1}{6(l+1)(l+2)}\left[(3 l+4) b_{l m}^{(2)}(X)-\frac{1}{4(l+1)} b_{l m}^{(0)}(X)\right],
\end{aligned}
$$

where $\bar{U}=(4 \pi)^{-1 / 2} U_{00}$. For $l=1$, the $p$-wave condition of Rassolov and Chipman [19] can be recovered. It also describes a case of two electrons in a triplet state. 


\section{HIGHER ORDER CUSP RELATIONS FOR THE DERIVATIVES OF THE PAIR DENSITY}

The pair density $n$ can be easily calculated from the wave function (10) by integrating $|\Phi|^{2}$ for all coordinates, except $\mathbf{r}_{1}$ and $\mathbf{r}_{2}$ :

$$
\begin{aligned}
& n\left(\mathbf{r}_{1}, \mathbf{r}_{2}\right) \\
& =\frac{N(N-1)}{2} \int\left|\Phi\left(\mathbf{r}_{1}, \sigma_{1}, \mathbf{r}_{2}, \sigma_{2}, \mathbf{r}_{3}, \sigma_{3}, \ldots, \mathbf{r}_{N}, \sigma_{N}\right)\right|^{2} \\
& \quad \times d \sigma_{1} d \sigma_{2} d \mathbf{r}_{3} d \sigma_{3} \cdots d \mathbf{r}_{N} d \sigma_{N} .
\end{aligned}
$$

Now $n$ is averaged for the polar angles $\hat{r}_{1}$ :

$$
\bar{n}\left(r_{1}, \mathbf{r}_{2}\right)=\frac{1}{4 \pi} \int d \hat{r}_{1} n\left(r_{1}, \hat{r}_{1}, \mathbf{r}_{2}\right) .
$$

Substituting Eq. (10) into Eq. (31), we obtain

$$
\bar{n}\left(r_{1}, \mathbf{r}_{2}\right)=\sum_{m} r_{1}^{2 l} \tilde{\chi}_{l m}\left(r_{1}, \mathbf{r}_{2}\right)^{2}+\sum_{m, l^{\prime}, m^{\prime} ; l^{\prime}>l} r_{1}^{2 l^{\prime}} \tilde{\chi}_{l^{\prime} m^{\prime}}\left(r_{1}, \mathbf{r}_{2}\right)^{2},
$$

where $\tilde{\chi}_{l m}^{2}$ is obtained after integrating $\chi_{l m}^{2}$ for the coordinates of $N-2$ electrons:

$$
\tilde{\chi}_{l m}^{2}=\frac{N(N-1)}{2} \int \chi_{l m}^{2} d Q
$$

$Q$ stands for all the coordinates of $N-2$ electrons. For highly excited states, the functions $\eta_{l}\left(r_{1}, \mathbf{r}_{2}\right)$ are introduced with the definition

$$
\eta_{l}\left(r_{1}, \mathbf{r}_{2}\right)=\frac{\bar{n}\left(r_{1}, \mathbf{r}_{2}\right)}{r_{1}^{2 l}}
$$

Equations (32) and (34) lead to

$$
\eta_{l}=\sum_{m} \tilde{\chi}_{l m}^{2}+\sum_{l^{\prime}, m^{\prime} ; l^{\prime}>l} r_{1}^{2\left(l^{\prime}-l\right)} \tilde{\chi}_{l^{\prime} m^{\prime}}^{2}
$$

For $r_{1}=0, \eta_{l}$ takes the form

$$
\eta_{l}\left(0, \mathbf{r}_{2}\right)=\sum_{m} \tilde{\chi}_{l m}^{2}\left(0, \mathbf{r}_{2}\right)=\frac{N(N-1)}{2} \sum_{m} \int\left[a_{l m}^{0}\right]^{2} d Q .
$$

Differentiating Eq. (35) with respect to $r_{1}$ and making use of Eq. (15), we arrive at

$$
\left.\frac{d}{d r_{1}} \eta_{l}\left(r_{1}, \mathbf{r}_{2}\right)\right|_{r_{1}=0}=-2 \frac{Z_{\alpha}}{l+1} \eta_{l}\left(0, \mathbf{r}_{2}\right) .
$$

The second derivative can be expressed as

$$
\begin{aligned}
& \left.\frac{d^{2}}{d r_{1}^{2}} \eta_{l}\left(r_{1}, \mathbf{r}_{2}\right)\right|_{r_{1}=0} \\
& =2\left(\frac { 1 } { 2 l + 3 } \left\{\left[\frac{Z_{\alpha}^{2}}{(l+1)^{2}}(4 l+5)-2 E\right] \eta_{l}\left(0, \mathbf{r}_{2}\right)\right.\right. \\
& \left.\left.\quad+2\left[W_{l}\left(0, \mathbf{r}_{2}\right)+G_{l}\left(0, \mathbf{r}_{2}\right)\right]\right\}+\eta_{l+1}\left(0, \mathbf{r}_{2}\right)\right),
\end{aligned}
$$

where

$$
W_{l}\left(0, \mathbf{r}_{2}\right)=\frac{N(N-1)}{2} \sum_{m} \int a_{l m}^{0} \bar{W} a_{l m}^{0} d Q
$$

$$
G_{l}\left(0, \mathbf{r}_{2}\right)=\frac{N(N-1)}{2} \sum_{m} \int a_{l m}^{0} \hat{G} a_{l m}^{0} d Q .
$$

In Eq. (38), we used the notation

$$
\eta_{l+1}(0)=\frac{N(N-1)}{2} \sum_{m} \int d Q\left|a_{l+1 m}^{(0)}(X)\right|^{2} .
$$

This notation means that there is a contribution from the zeroorder part of $\chi_{l+1}$. Thus, while the first derivative of $\eta_{l}$ at the cusp can be expressed with the atomic number of the nucleus considered and the value of $\eta$ at the cusp, the second derivative includes the energy, the potential at the nucleus, the integral of the operator $G$, and the $\eta_{l+1}(0)$, too. So there is a contribution from the $l+1$ terms.

The third derivative of $\eta_{l}$ [Eq. (35)] takes the form

$$
\begin{aligned}
\frac{d^{3}}{d r_{1}^{3}} & \left.\eta_{l}\left(r_{1}, \mathbf{r}_{2}\right)\right|_{r_{1}=0} \\
= & \frac{4 Z_{\alpha}}{(l+1)(l+2)(2 l+3)}\left(2 ( 3 l + 5 ) \left\{E \eta_{l}\left(0, \mathbf{r}_{2}\right)\right.\right. \\
& \left.\left.-\left[W_{l}\left(0, \mathbf{r}_{2}\right)+G_{l}\left(0, \mathbf{r}_{2}\right)\right]\right\}-\frac{4 l+7}{l+1} Z_{\alpha}^{2} \eta_{l}\left(0, \mathbf{r}_{2}\right)\right) \\
& -12 \frac{Z_{\alpha}}{l+2} \eta_{l+1}\left(0, \mathbf{r}_{2}\right) .
\end{aligned}
$$

From Eqs. (37)-(42), we arrive at a relation between the function $\eta$ and its first and third derivatives:

$$
\begin{aligned}
\eta_{l}^{\prime \prime \prime}\left(0, \mathbf{r}_{2}\right)= & \frac{2 Z_{\alpha}}{(l+1)(l+2)}\left[-(3 l+5) \eta_{l}^{\prime \prime}\left(0, \mathbf{r}_{2}\right)\right. \\
& \left.+\frac{4(2 l+3) Z_{\alpha}^{2}}{(l+1)^{2}} \eta_{l}\left(0, \mathbf{r}_{2}\right)+4 \eta_{l+1}\left(0, \mathbf{r}_{2}\right)\right]
\end{aligned}
$$

Equation (43) gives the dependence of the third derivative of the pair density at the nucleus on the lower derivatives.

Now we turn to the electron-electron cusp relations. With the definition

$$
\xi_{l}\left(r_{12}, \mathbf{R}\right)=\frac{\bar{n}\left(r_{12}, \mathbf{R}\right)}{r_{12}^{2 l}},
$$

and using Eq. (27), we obtain

$$
\xi_{l}(0, \mathbf{R})=\sum_{m} \tilde{\chi}_{l m}^{2}(0, \mathbf{R})=\frac{N(N-1)}{2} \sum_{m} \int\left[b_{l m}^{0}\right]^{2} d Q .
$$

The derivative of Eq. (44) with respect to $r_{12}$ leads to

$$
\left.\frac{d}{d r_{12}} \xi_{l}\left(r_{12}, \mathbf{R}\right)\right|_{r_{12}=0}=\frac{1}{l+1} \xi_{l}(0, \mathbf{R}) .
$$

The second derivative can be expressed as

$$
\begin{aligned}
& \left.\frac{d^{2}}{d r_{12}^{2}} \xi_{l}\left(r_{12}, \mathbf{R}\right)\right|_{r_{12}=0} \\
& \quad=2\left(\frac { 1 } { 2 l + 3 } \left\{\left[\frac{4 l+5}{(l+1)^{2}}-E\right] \xi_{l}(0, \mathbf{R})\right.\right. \\
& \left.\left.\quad+\left[U_{l}(0, \mathbf{R})+K_{l}(0, \mathbf{R})\right]\right\}+\xi_{l+1}(0, \mathbf{R})\right),
\end{aligned}
$$


where

$$
\begin{aligned}
& U_{l}(0, \mathbf{R})=\frac{N(N-1)}{2} \sum_{m} \int b_{l m}^{0} \bar{U} b_{l m}^{0} d Q, \\
& K_{l}(0, \mathbf{R})=\frac{N(N-1)}{2} \sum_{m} \int b_{l m}^{0} \hat{K} b_{l m}^{0} d Q .
\end{aligned}
$$

The third derivative takes the form

$$
\begin{aligned}
\frac{d^{3}}{d r_{12}^{3}} & \left.\xi_{l}\left(r_{12}, \mathbf{R}\right)\right|_{r_{12}=0} \\
= & -\frac{2}{(l+1)(l+2)(2 l+3)}\left(( 3 l + 5 ) \left\{E \xi_{l}(0, \mathbf{R})\right.\right. \\
& \left.\left.-\left[U_{l}(0, \mathbf{R})+K_{l}(0, \mathbf{R})\right]\right\}-\frac{4 l+7}{4(l+1)} \xi_{l}(0, \mathbf{R})\right) \\
& +\frac{6}{l+2} \xi_{l+1}(0, \mathbf{R}) .
\end{aligned}
$$

Equations (45)-(47) and (50) lead to a relation between the function $\eta$ and its first and third derivatives:

$$
\begin{aligned}
\left.\frac{d^{3}}{d r_{12}^{3}} \xi_{l}\left(r_{12}, \mathbf{R}\right)\right|_{r_{12}=0} & \frac{1}{=} \frac{1}{(l+1)(l+2)}\left[\left.(3 l+5) \frac{d^{2}}{d r_{12}^{2}} \xi_{l}\left(r_{12}, \mathbf{R}\right)\right|_{r_{12}=0}\right. \\
& \left.-\frac{2 l+3}{(l+1)^{2}} \xi_{l}(0, \mathbf{R})-4 \xi_{l+1}(0, \mathbf{R})\right] .
\end{aligned}
$$

\section{ILLUSTRATIVE EXAMPLES AND DISCUSSION}

The cusp relations derived earlier are now illustrated with few simple examples. Consider first two electrons in a bare nuclear field with charge $Z$. The electrons are in the $2 p(l=1$, $m=0)$ excited state with antiparallel spins. Then the spatial part of the wave function is symmetric:

$$
\Psi\left(\mathbf{r}_{1}, \mathbf{r}_{2}\right)=\phi_{2 p}\left(\mathbf{r}_{1}\right) \phi_{2 p}\left(\mathbf{r}_{2}\right),
$$

where

$$
\phi_{2 p}(\mathbf{r})=\operatorname{cr} e^{-Z r / 2} Y_{1 m}(\Omega),
$$

where $c$ is a constant. We can readily obtain the pair density average for the polar angles $\Omega_{1}$ :

$$
\bar{n}\left(r_{1}, \mathbf{r}_{2}\right)=f\left(\mathbf{r}_{2}\right) r_{1}^{2} e^{-Z r_{1}}
$$

where

$$
f\left(\mathbf{r}_{2}\right)=c^{4} r_{2}^{2} e^{-Z r_{2}}\left|Y_{1 m}\left(\Omega_{2}\right)\right|^{2} \frac{1}{4 \pi} .
$$

Then we define the function $\eta_{1}$ as

$$
\eta_{1}\left(r_{1}, \mathbf{r}_{2}\right)=\frac{\bar{n}\left(r_{1}, \mathbf{r}_{2}\right)}{r_{1}^{2}}
$$

Equations (54) and (56) lead to the result

$$
\eta_{1}\left(r_{1}, \mathbf{r}_{2}\right)=f\left(\mathbf{r}_{2}\right) e^{-Z r_{1}} .
$$

Consequently,

$$
\eta_{1}\left(0, \mathbf{r}_{2}\right)=f\left(\mathbf{r}_{2}\right)
$$

We can immediately calculate the first derivative of the function $\eta_{1}$ and take its value at $r_{1}=0$ :

$$
\left.\frac{d}{d r_{1}} \eta_{1}\left(r_{1}, \mathbf{r}_{2}\right)\right|_{r_{1}=0}=-Z f\left(\mathbf{r}_{2}\right)=-Z \eta_{1}\left(0, \mathbf{r}_{2}\right),
$$

with complete agreement with Eq. (37) as $l=1$. The second and third derivatives at $r_{1}=0$ are as follows:

$$
\begin{gathered}
\left.\frac{d^{2}}{d r_{1}^{2}} \eta_{1}\left(r_{1}, \mathbf{r}_{2}\right)\right|_{r_{1}=0}=Z^{2} f\left(\mathbf{r}_{2}\right)=Z^{2} \eta_{1}\left(0, \mathbf{r}_{2}\right), \\
\left.\frac{d^{3}}{d r_{1}^{3}} \eta_{1}\left(r_{1}, \mathbf{r}_{2}\right)\right|_{r_{1}=0}=-Z^{3} f\left(\mathbf{r}_{2}\right)=-Z^{3} \eta_{1}\left(0, \mathbf{r}_{2}\right) .
\end{gathered}
$$

We can readily check that Eqs. (58)-(61) satisfy Eq. (43).

As a second example, consider two electrons confined by a harmonic potential [34]. This harmonium (or Hooke's atom) is described by the Hamiltonian

$$
\hat{H}=-\frac{1}{2} \nabla_{1}^{2}+\frac{1}{2} \omega^{2} r_{1}^{2}-\frac{1}{2} \nabla_{2}^{2}+\frac{1}{2} \omega^{2} r_{2}^{2}+\frac{1}{r_{12}} .
$$

After introducing the intracular and extracular coordinates [Eq. (22)], we immediately notice that the center-of-mass equation is the well-known three-dimensional oscillator solution. The relative motion of the two electrons is characterized by the Schrödinger equation:

$$
\left[-\frac{1}{2} \nabla_{12}^{2}+\frac{1}{2} \omega_{r}^{2} r_{12}^{2}+\frac{1}{2 r_{12}}\right] \phi\left(r_{12}\right)=\varepsilon^{\prime} \phi\left(r_{12}\right),
$$

where $\omega_{r}=\omega / 2$ and $\varepsilon^{\prime}=\omega[2(l+n)+1] / 4$. An analytical solution can be given, for example, for $n=2$ and arbitrary $l$ with the energy $\varepsilon^{\prime}=(2 l+5) /[8(l+1)]$. The electrons have antiparallel spins. Then the spatial part of the wave function is symmetric:

$$
\Psi\left(\mathbf{r}_{12}, \mathbf{R}\right)=\phi\left(\mathbf{r}_{12}\right) \zeta(\mathbf{R}),
$$

where $\zeta(\mathbf{R})$ is the wave function of the center-of-mass motion. The wave function of the relative motion then takes the form

$$
\phi\left(r_{12}\right)=\frac{u\left(r_{12}\right)}{r_{12}} Y_{l m}\left(\widehat{r_{12}}\right)
$$

where

$$
u\left(r_{12}\right)=r_{12}^{l+1}\left[1+\frac{r_{12}}{2(l+1)}\right] e^{-\frac{r_{12}^{2}}{8(l+1)}} .
$$

Making use of Eq. (31), we obtain

$$
\bar{n}\left(r_{1}, \mathbf{r}_{2}\right)=r_{12}^{2 l}\left[1+\frac{r_{12}}{2(l+1)}\right]^{2} e^{-\frac{r_{12}^{2}}{4(l+1)}} \frac{|\zeta(\mathbf{R})|^{2}}{4 \pi} .
$$

Then we define the function $\xi_{1}$ as

$$
\xi_{l}\left(r_{12}, \mathbf{R}\right)=\frac{\bar{n}\left(r_{12}, \mathbf{R}\right)}{r_{12}^{2 l}}
$$

Equations (67) and (68) lead to the result

$$
\xi_{l}\left(r_{12}, \mathbf{R}\right)=\left[1+\frac{r_{12}}{2(l+1)}\right]^{2} e^{-\frac{r_{12}^{2}}{4(l+1)}} \frac{|\zeta(\mathbf{R})|^{2}}{4 \pi} .
$$


At the electron-electron coalescence

$$
\xi_{l}(0, \mathbf{R})=\frac{|\zeta(\mathbf{R})|^{2}}{4 \pi}
$$

we can immediately calculate the first derivative of the function $\xi_{l}$ and take its value at $r_{12}=0$ :

$$
\left.\frac{d}{d r_{12}} \xi_{l}\left(r_{12}, \mathbf{R}\right)\right|_{r_{12}=0}=\frac{|\zeta(\mathbf{R})|^{2}}{4 \pi} \frac{1}{l+1}=\frac{\xi_{l}(0, \mathbf{R})}{l+1},
$$

with complete agreement with Eq. (46). The second and third derivatives at $r_{12}=0$ are as follows:

$$
\begin{aligned}
& \left.\frac{d^{2}}{d r_{12}^{2}} \xi_{l}\left(r_{12}, \mathbf{R}\right)\right|_{r_{12}=0}=-\frac{|\zeta(\mathbf{R})|^{2}}{4 \pi} \frac{l}{2(l+1)^{2}}, \\
& \left.\frac{d^{3}}{d r_{12}^{3}} \xi_{l}\left(r_{12}, \mathbf{R}\right)\right|_{r_{12}=0}=-\frac{|\zeta(\mathbf{R})|^{2}}{4 \pi} \frac{3}{2(l+1)^{2}} .
\end{aligned}
$$

We can readily check that Eqs. (70)-(73) satisfy Eq. (51).

Note that in both examples, the term $\eta_{l+1}=0$ in Eqs. (43) and (51). This is the consequence of the fact that both electrons have the same azimuthal quantum number $l$. In these examples, we can see the importance of the generalization of the relations for the values of $l>0$.

As a further example, we could take three electrons in a bare Coulomb potential $-Z / r$. Suppose we have an excited state of symmetry ${ }^{4} S_{u}$ for a configuration $2 p^{3}$, the spatial part of the wave function is factorized, and we have

$$
\Psi(1,2,3)=\frac{1}{\sqrt{6}}\left|\begin{array}{lll}
\phi_{1}(1) & \phi_{1}(2) & \phi_{1}(3) \\
\phi_{2}(1) & \phi_{2}(2) & \phi_{2}(3) \\
\phi_{3}(1) & \phi_{3}(2) & \phi_{3}(3)
\end{array}\right|,
$$

where the $\phi_{j}$ are H-like $2 p$ orbitals with $m=0, \pm 1$. The pair density is

$$
\begin{aligned}
n(1,2)= & \frac{1}{2}\left[\phi_{1}(1)^{2} \phi_{2}(2)^{2}+\phi_{2}(1)^{2} \phi_{1}(2)^{2}+\phi_{1}(1)^{2} \phi_{3}(2)^{2}\right. \\
& +\phi_{3}(1)^{2} \phi_{1}(2)^{2}+\phi_{2}(1)^{2} \phi_{3}(2)^{2}+\phi_{3}(1)^{2} \phi_{2}(2)^{2} \\
& -2\left(\phi_{1} \phi_{2}\right)(1)\left(\phi_{1} \phi_{2}\right)(2)-2\left(\phi_{1} \phi_{3}\right)(1)\left(\phi_{1} \phi_{3}\right)(2) \\
& \left.-2\left(\phi_{2} \phi_{3}\right)(1)\left(\phi_{2} \phi_{3}\right)(2)\right] .
\end{aligned}
$$

Finally, we can average over the polar angle $\Omega_{1}$, getting

$$
\begin{aligned}
& \bar{n}\left(r_{1}, \mathbf{r}_{2}\right) \propto r_{1}^{2} e^{-Z r_{1}}\left[\phi_{1}\left(\mathbf{r}_{2}\right)^{2}+\phi_{2}\left(\mathbf{r}_{2}\right)^{2}+\phi_{3}\left(\mathbf{r}_{2}\right)^{2}\right] \\
& \quad=c r_{1}^{2} r_{2}^{2} e^{-Z\left(r_{1}+r_{2}\right)},
\end{aligned}
$$

and the cusp conditions are easily verified.

The pair density is a very important quantity. It has a fundamental role in describing electron systems. Contrary to the electron density, it is not easy to give adequate approximation to the pair density. Exact relations for the pair density can be helpful to judge the quality of an approximation. The cusp relations presented earlier have the charming feature that they are valid not only for the ground state but for arbitrary excited states, too.

The pair density is the key quantity of the pair density functional theory. It was shown $[21,22]$ that the problem of an arbitrary system with even electrons can be reduced to a twoparticle problem. This means an enormous simplification as we always have to solve a two-particle equation independently of the number of electrons. This remarkable result has the drawback that we do not know the exact form of the Pauli potential. Exact relations and theorems play a very important role in the density functional theory as they have proved useful in improving the accuracy of approximate energy functionals. We believe that these relations are also very useful in the pair density functional theory. We have proved [21,22] that the unknown Pauli potential can be constructed from the pair density. Therefore these exact relations might be useful in inventing accurate approximations for the Pauli potential.

In conclusion, it is stated that there exist higher order cusp relations for the pair density. These relations are derived both for the electron-nucleus and the electron-electron coalescences, and they are valid both for the ground and the excited states of atoms, ions, or molecules.

\section{ACKNOWLEDGMENTS}

This article was written in the frame of the Bilateral Scientific Cooperation between Italy and Hungary, sponsored by Consiglio Nazionale delle Ricerche and the Hungarian Academy of Sciences. Grant OTKA No. K 67923 is gratefully acknowledged.
[1] T. Kato, Commun. Pure Appl. Math. 10, 151 (1957).

[2] W. Kolos and C. C. J. Roothaan, Rev. Mod. Phys. 32, 205 (1960).

[3] C. C. J. Roothaan and P. S. Kelly, Phys. Rev. 131, 1177 (1963).

[4] R. T. Pack and W. B. Brown, J. Chem. Phys. 45, 556 (1966).

[5] E. Steiner, J. Chem. Phys. 39, 2365 (1963); N. H. March, SelfConsistent Fields in Atoms (Pergamon, Oxford, 1975).

[6] W. A. Bingel, Z. Naturforsch. Teil A 18, 1249 (1963).

[7] J. C. Kimball, Phys. Rev. A 7, 1648 (1973); J. Phys. A 8, 1513 (1975).

[8] A. K. Rajagopal, J. C. Kimball, and M. Banerjee, Phys. Rev. B 18, 2339 (1978).

[9] E. R. Davidson, Reduced Density Matrices in Quantum Chemistry (Academic, New York, 1976).

[10] D. P. Tew, J. Chem. Phys. 129, 014104 (2008).

[11] B. R. Johnson, Phys. Rev. A 24, 2339 (1981).
[12] C. R. Myers, C. J. Umrigar, J. P. Sethna, and J. D. Morgan III, Phys. Rev. A 44, 5537 (1991).

[13] S. Fournais, M. Hoffmann-Ostenhof, T. Hoffmann-Ostenhof, and T. Ostergard Sorensen, Commun. Math. Phys. 255, 183 (2005).

[14] Á. Nagy and K. D. Sen, J. Phys. B 33, 1745 (2000).

[15] R. O. Esquivel, J. Chen, M. J. Stott, R. P. Sagar, and V. H. Smith Jr., Phys. Rev. A 47, 936 (1993).

[16] R. O. Esquivel, R. P. Sagar, V. H. Smith Jr., J. Chen, and M. J. Stott, Phys. Rev. A 47, 4735 (1993).

[17] Á. Nagy and K. D. Sen, Chem. Phys. Lett. 332, 154 (2000).

[18] N. H. March, I. A. Howard, A. Holas, P. Senet, and V. E. Van Doren, Phys. Rev. A 63, 012520 (2000).

[19] V. A. Rassolov and D. M. Chipman, J. Chem. Phys. 104, 9908 (1996). 
[20] Á. Nagy and K. D. Sen, J. Chem. Phys. 115, 6300 (2001).

[21] Á. Nagy, Phys. Rev. A 66, 022505 (2002).

[22] Á. Nagy, in The Fundamentals of Electron Density, Density Matrices and Density Functional Theory in Atoms, Molecules and Solid State, edited by N. I. Gidopoulos and S. Wilson (Kluwer, New York, 2003), p. 79.

[23] Á. Nagy and C. Amovilli, J. Chem. Phys. 121, 6640 (2004).

[24] Á. Nagy, J. Chem. Phys. 125, 184104 (2006).

[25] F. Furche, Phys. Rev. A 70, 022514 (2004).

[26] Á. Nagy and C. Amovilli, J. Chem. Phys. 128, 114115 (2008).

[27] Á. Nagy and C. Amovilli, Chem. Phys. Lett. 469, 353 (2009).

[28] P. Ziesche, Phys. Lett. A 195, 195 (1994); Int. J. Quantum Chem. 60, 149 (1996).
[29] M. Higuchi and K. Higuchi, Phys. B 387, 117 (2007).

[30] M. Higuchi, M. Miyasta, M. Kodera, and K. Higuchi, J. Phys. Cond. Matt. 19, 365219 (2007).

[31] P. W. Ayers and M. Levy, Chem. Phys. Lett. 415, 211 (2005).

[32] P. W. Ayers and M. Levy, J. Chem. Sci. 117, 507 (2006).

[33] E. J. Knystautas, J. Phys. B 25, L395 (1992); Phys. Rev. Lett. 69, 2635 (1992); M. Agentoft, T. Andersen, and K. T. Chung, J. Phys. B 17, L433 (1984); S. Mannervik, R. T. Short, D. Sonnek, E. Träbert, G. Möller, V. Lodwig, P. H. Heckmann, J. H. Blanke, and K. Brand, Phys. Rev. A 39, 3964 (1989); E. Träbert, P. H. Heckmann, J. Doerfert, and J. Granzow, J. Phys. B 25, L353 (1992).

[34] M. Taut, Phys. Rev. A 48, 3561 (1993); J. Phys. A 27, 1045 (1994). 